\title{
Effects of a Perpendicularly Applied Magnetic Field on Harmonically Driven Quasi-two-dimensional Electron Gas: the Static Macrostates Symmetry Breaking and Generation of Even Harmonics in System Output
}

\section{I. Maglevanny， V. A. Smolar，T. I. Karyakina}

In this paper, we consider activation processes in a nonlinear metastable system based on a quasi-two-dimensional superlattice and study the dynamics of such a system, which is externally driven by a harmonic force in regimes of controlled instabilities. The spontaneous transverse electric field is considered as an order parameter and the forced violations of the order parameter are considered as a response of a system to periodic driving. The internal control parameters are the longitudinal applied electric field, the sample temperature and the magnetic field which is orthogonal to the superlattice plane. We investigate the cooperative effects of self-organization and high harmonic forcing in such a system from the viewpoint of catastrophe theory It is shown through numerical simulations that the additional magnetic field breaks the static macrostates symmetry and leads to generation of even harmonics; it also allows the control of the intensity of particular harmonics. The intensity of even harmonics demonstrates resonanttype nonmonotonic dependence on control parameters with the maxima at points close to critical points of the synergetic potential.

Keywords: lateral superlattices, bifurcation-based device, spontaneous transverse electric field, nonequilibrium phase transitions, symmetry breaking of magnetic-field-induced macrostates, resonant enhancement of even harmonics

Received January 30, 2021

Accepted June 01, 2021

Ilya I. Maglevanny

sianko@list.ru

Tatyana I. Karyakina

karyakina@fizmat.vspu.ru

Volgograd State Socio-Pedagogical University

prosp. Lenina 27, Volgograd, 400066 Russia

Vladimir A. Smolar

smolar.v@yandex.ru

Volgograd State Technical University

prosp. Lenina 28, Volgograd, 400131 Russia

RUSSIAN JOURNAL OF NONLINEAR DYNAMICS, 2021, 17(2), 141-156 


\section{Introduction}

In solving applied and research problems, there often arise situations where the number of stable states of the system, or the character of motion, change with varying parameters. In practice, problems where bifurcations take place are of interest. Bifurcation is usually understood as a qualitative change in the behavior of a system where the number of stable states of the system, or the character of motion, change with smooth change of parameters [1, 2]. Modeling of nonlinear dynamics near bifurcation points is particularly interesting [3].

The influence of harmonic forcing on nonlinear dynamical systems and the analysis of forced vibrations as a response to periodic driving is a major tool for characterizing physical systems and is a very important area of research.

An important feature of signal transformation through a nonlinear filtering system is vibration nonlinearity, which becomes significant even at small vibration amplitudes. In the vicinity of bifurcation points, where the external forcing has its greatest influence, the system goes to the state with either large or small output, depending on the input signal. It allows investigating a broad variety of nonlinear phenomena, including bifurcations and constructing of bifurcationbased devices.

With the development of heterostructures, the subject of the nonlinear carrier transport of low-dimensional carrier gas is growing and becoming more and more important. Semiconductor superlattices have been at the focal point of research in the last few decades because of their unique highly nonlinear electronic properties. These materials have a promising potential chiefly because they provide a novel platform for fundamental science studies and a diverse and unusual array of physical properties that can be employed in practical applications.

The artificially grown quasi-two-dimensional structure - the lateral superlattice $(2 \mathrm{SL})[4,5]$ may serve as a rather suitable model system for investigation of self-organization of quasi-twodimensional electron gas [6-8] and nonlinear signal transfer $[9,10]$ in complex nonlinear systems.

In the present work we consider the influence of a magnetic field (under Hall-measurement conditions) on the output of a harmonically driven system, based on lateral 2SL. We consider the nonequilibrium electron gas from the viewpoint of catastrophe theory (which provides a powerful tool to discover a criterion for stability and instability in a given system in a situation where the nonequilibrium phase transitions (NPTs) occur) and show that the magnetic field violates the symmetry of synergetic potential and leads to violation of static macrostates resulting in multiple hysteresis regimes and to appearance of even harmonics in the system output through the breaking of half-wave symmetry of the output signal.

\section{Sample configuration and calculation of static current density}

Before the novel signal detection strategy is proposed, a criterion for evaluating the output signal and system performance is essential, because all the parameters need to be optimized for the best matching system and optimal output. In this context the interconnections of elements of the structure under study and the choice of order and control parameters should be defined.

In this paper we consider the following model scheme, presented in Fig. 1, where 2SL is a nonlinear load.

The effects considered in this paper occur when the applied electric field makes an angle $\alpha=45^{\circ}$ with the principal [01] — axis (Fig. 1). This direction is defined as the $O X$ - axis. The sample is supposed to be closed-circuited in series with a finite resistor $R$ in the $O Y$ direction. 


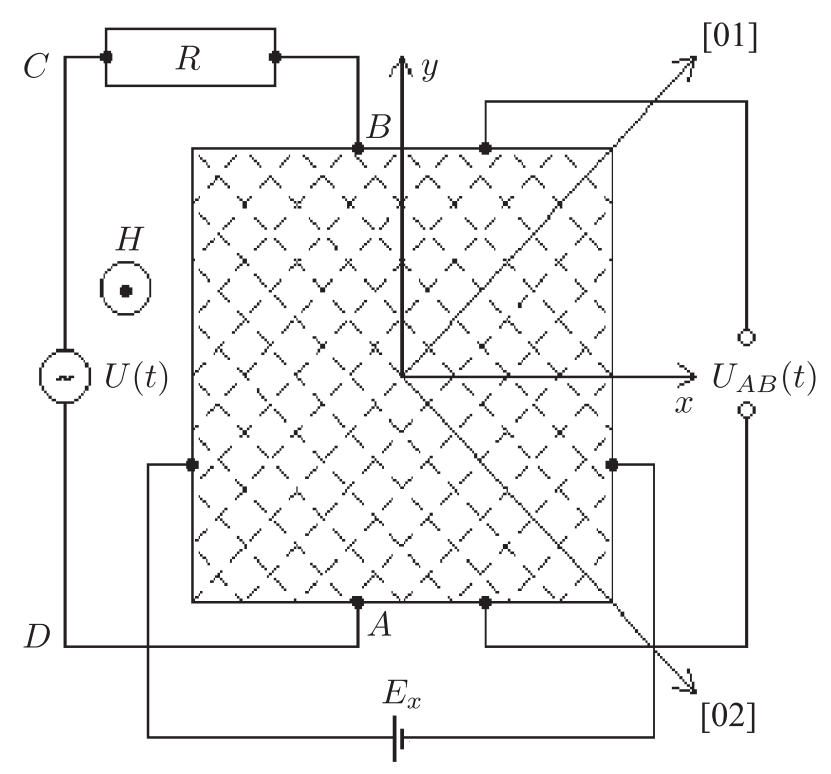

Fig. 1. Scheme of inclusion of the magnetic field and of the applied electric field $E_{x}$ in the 2SL sample under the harmonic forcing $U(t)=U_{0} \cos \Omega t$.

The external electric field $\left(E_{x}\right)$ is applied to the 2SL sample and the magnetic field $\boldsymbol{H}=$ $=(0,0, H) \| O Z$ is applied normally to the $2 \mathrm{SL}$ surface $(x y)$. These fields give rise to a transverse field $E_{y}$ which is treated as an order parameter.

The system is driven by an a priori known harmonic signal $U(t)=U_{0} \cos \Omega t$ (Fig. 1). The harmonic forcing defines the output signal $U_{A B}(t)=L E_{y}(t)$ - the response of the system, where $L$ is the sample length in the $O Y$ direction. The system control parameters are $R, E_{x}, H$ and $T$, where $T$ is the sample temperature.

For further calculation the static current density in 2SL under given conditions should be defined. When one uses a tight-binding approximation, the energy band is confined to two directions $[4,5]$ and in the reference frame defined in Fig. 1 the energy spectrum takes the form $[9,10]$

$$
\varepsilon(\boldsymbol{p})=\Delta-\Delta \cos \frac{p_{x} d}{\hbar} \cos \frac{p_{y} d}{\hbar} \quad\left(d=\frac{a}{\sqrt{2}}\right),
$$

where $2 \Delta$ is the allowed miniband width, $a$ is the SL constant, and $p_{x}$ and $p_{y}$ are the components of the carrier crystal momentum $(\boldsymbol{p})$ with respect to axes $O x$ and $O y$ of the $2 \mathrm{SL}$ which are shown in Fig. 1. The motion of the electron along the third direction is considered to be "frozen".

To calculate the current density ( $\boldsymbol{J}$ ) originated from carriers with the dispersion law (2.1), we confine ourselves to the semiclassical $(2 \Delta \gg \hbar / \tau ; e|\boldsymbol{E}| d)$ and single-band approximation $\left(e|\boldsymbol{E}| d \ll \varepsilon_{g}\right)$, where $\varepsilon_{g}$ is the forbidden miniband width and $\tau$ is the mean free time of electrons. For the magnetic field we assume $e|\boldsymbol{H}| \Delta d^{2} / c \hbar \ll k T$, i.e., it is nonquantizing ( $k$ is the Boltzmann constant).

To present the results in dimensionless form, we introduce the measurement units $p_{0}=\hbar / d$, $T_{0}=\Delta / k, E_{0}=\hbar / e d \tau, H_{0}=\hbar^{2} c / e \tau \Delta d^{2}, j_{0}=n e \Delta d / \hbar$ ( $n$ is the carrier density) and change the notation by writing

$$
\frac{t}{\tau} \rightarrow t, \quad \frac{\boldsymbol{p}}{p_{0}} \rightarrow \boldsymbol{p}, \quad \frac{T}{T_{0}} \rightarrow T, \quad \frac{\boldsymbol{E}}{E_{0}} \rightarrow \boldsymbol{E}, \quad \frac{\boldsymbol{H}}{H_{0}} \rightarrow \boldsymbol{H}, \quad \frac{\boldsymbol{\jmath}}{j_{0}} \rightarrow \boldsymbol{\jmath} .
$$


The necessary charge motion equation may be represented as

$$
\frac{\mathrm{d} \boldsymbol{p}}{\mathrm{d} t}=\boldsymbol{E}+\boldsymbol{v}(\boldsymbol{p}) \times \boldsymbol{H}, \quad \boldsymbol{p}(0)=0, \quad \boldsymbol{v}=\frac{1}{\Delta} \frac{\partial \varepsilon(\boldsymbol{p})}{\partial \boldsymbol{p}},
$$

where $\varepsilon(\boldsymbol{p})=\Delta\left(1-\cos p_{x} \cos p_{y}\right)$ and $\boldsymbol{v}$ is is the dimensionless charge velocity

$$
v_{x}(\boldsymbol{p})=\sin p_{x} \cos p_{y}, \quad v_{y}(\boldsymbol{p})=\cos p_{x} \sin p_{y}, \quad v_{z}(\boldsymbol{p})=0 .
$$

We use the Boltzmann kinetic equation with the $\tau=$ const approximation of the collision integral (one of the arguments in favor of such an approximation are the results of [11] in which it has been established experimentally that at $T>40 \mathrm{~K}$ the mean free time $\tau=$ const and does almost not depend on temperature). The equation for the nonequilibrium distribution function is

$$
\frac{\partial f}{\partial t}+\frac{\mathrm{d} \boldsymbol{p}}{\mathrm{d} t} \cdot \frac{\partial f}{\partial \boldsymbol{p}}=f_{0}(\boldsymbol{p})-f
$$

Here $f_{0}(\boldsymbol{p})$ is the equilibrium distribution function.

Then the steady-state solution $(\partial f / \partial t=0)$ of Eq. (2.5) can be represented in the form [12]

$$
f(\boldsymbol{p}, \boldsymbol{E}, \boldsymbol{H})=\int_{0}^{\infty} f_{0}(\boldsymbol{p}-\boldsymbol{p}(t, \boldsymbol{E}, \boldsymbol{H})) e^{-t} d t
$$

where $\boldsymbol{p}(t, \boldsymbol{E}, \boldsymbol{H})$ is a solution of the Cauchy problem (2.3).

The current density of charge carriers is defined as [12]

$$
\boldsymbol{\jmath}=\frac{1}{n} \sum_{\boldsymbol{p}} f_{0}(\boldsymbol{p}) \int_{0}^{\infty} \boldsymbol{v}(\boldsymbol{p}+\boldsymbol{p}(t)) e^{-t} d t
$$

where the summation is made within the first Brillouin zone.

Substituting (2.4) into (2.7), we finally get $[8,9]$

$$
\boldsymbol{\jmath}(\boldsymbol{E}, \boldsymbol{H}, T)=C(T) \int_{0}^{\infty} \boldsymbol{v}(\boldsymbol{p}(t, \boldsymbol{E}, \boldsymbol{H})) e^{-t} d t
$$

and $C(T)=\left\langle\cos p_{x} \cos p_{y}\right\rangle$. Here the angular brackets mean the averaging over the equilibrium carrier distribution

$$
C(T)=\frac{1}{n} \sum_{\boldsymbol{p}} \cos p_{x} \cos p_{y} f_{0}(\boldsymbol{p})=\frac{1}{n} \iint_{-\pi}^{\pi} \cos p_{x} \cos p_{y} f_{0}\left(p_{x}, p_{y}\right) d p_{x} d p_{y} .
$$

In the following we consider an electron gas that is nondegenerate

$$
f_{0}(\boldsymbol{p})=n \exp \left(-\frac{\varepsilon(\boldsymbol{p})}{T}\right) / \sum_{\boldsymbol{p}} \exp \left(-\frac{\varepsilon(\boldsymbol{p})}{T}\right)
$$


then $[8,9]$

$$
C(T)=\frac{\iint_{-\pi}^{\pi} \cos p_{x} \cos p_{y} \exp \left(\frac{\cos p_{x} \cos p_{y}}{T}\right) d p_{x} d p_{y}}{\iint_{-\pi}^{\pi} \exp \left(\frac{\cos p_{x} \cos p_{y}}{T}\right) d p_{x} d p_{y}}=\frac{I_{1}(0.5 / T)}{I_{0}(0.5 / T)},
$$

where $I_{n}(z)$ is the modified Bessel function. The function (2.11) is monotonically decreasing, and $\lim _{T \rightarrow 0+0} C(T)=1, \lim _{T \rightarrow+\infty} C(T)=0$.

At $|H| \ll 1$ the current density $\boldsymbol{\jmath}$, defined by (2.3) and (2.8), may be found analytically in linear approximation on $H[13,14]$. At high $H$ the calculations cannot be accomplished analytically and some computational problems had to be solved.

We include the calculation of $\boldsymbol{\jmath}$ and the value $\partial j_{y} / \partial E_{y}$ (needed for the Morce characteristic of a static macrostate) in the common scheme. For that we define additional functions $s(t)$, $\boldsymbol{q}(t)=\partial \boldsymbol{p} / \partial E_{y}, w(t)$ and consider the dynamical system

$$
\left\{\begin{array}{l}
\dot{p}_{x}=E_{x}+H \cos p_{x} \sin p_{y} \\
\dot{p}_{y}=E_{y}-H \cos p_{y} \sin p_{x} \\
\dot{s}_{x}=e^{-t} \cos p_{y} \sin p_{x} \\
\dot{s}_{y}=e^{-t} \cos p_{x} \sin p_{y} \\
\dot{q}_{x}=H\left(q_{y} \cos p_{x} \cos p_{y}-q_{x} \sin p_{x} \sin p_{y}\right) \\
\dot{q}_{y}=1+H\left(q_{y} \sin p_{x} \sin p_{y}-q_{x} \cos p_{x} \cos p_{y}\right) \\
\dot{w}=e^{-t}\left(q_{y} \cos p_{x} \cos p_{y}-q_{x} \sin p_{x} \sin p_{y}\right)
\end{array}\right.
$$

for which zero conditions at $t=0$ hold and $E_{x}, E_{y}, H$ are considered as parameters. Then $\boldsymbol{J}$ and $\partial j_{y} / \partial E_{y}$ may be defined as asymptotics

$$
\boldsymbol{\jmath}=C(T) \lim _{t \rightarrow+\infty} \boldsymbol{s}(t), \quad \frac{\partial j_{y}}{\partial E_{y}}=C(T) \lim _{t \rightarrow+\infty} w(t) .
$$

The presence of factor $e^{-t}$ guarantees fast convergence to asymptotic values.

This algorithm reduces the calculation to solving the Cauchy problem (2.13) on some segment $t \in\left[0, t_{\max }\right]$, the asymptotic values being evaluated at $t=t_{\max }$. For that the embedded fifth-order explicit Runge-Kutta rule with adapted step-size control based on the Dormand and Prince scheme $[15,16]$ was used and the necessary value of $t_{\max }$ to reach reliable accuracy was controlled.

\section{Order parameter dynamics and bifurcations of static macrostates}

Assume that the period of the modulation signal significantly exceeds the internal relaxation times of the system. Then the dynamics of the order parameter is determined by the second Kirchhoff law for the contour ABCDA for instant current values, which leads to equation $[9,10]$

$$
U_{0} \cos (\Omega t)=L E_{0} E_{y}+S R\left[\frac{\varepsilon}{4 \pi} \frac{\mathrm{d}\left(E_{0} E_{y}\right)}{\mathrm{d} t}+j_{0} j_{y}\right]
$$


Here the Gaussian system of units is used, $\varepsilon$ is the permittivity of the sample's material, $j_{y}$ is the normalized static current density in the transverse direction (the nonlinear function of the order parameter and control parameters), and $S$ is the section of the specimen with the plane $O X Z$.

To normalize further Eq. (3.1), we introduce the measurement units of conductivity $\sigma_{0}=j_{0} / E_{0}$, time $t_{0}=\varepsilon /\left(4 \pi \sigma_{0}\right)$ and frequency $\Omega_{0}=1 / t_{0}$ and change the notation by writing

$$
\frac{t}{t_{0}} \Rightarrow t, \quad \frac{\Omega}{\Omega_{0}} \Rightarrow \Omega
$$

As a result, we get the normalized equation which defines the forced dynamics of the order parameter in standard gradient form

$$
\frac{\mathrm{d} E_{y}}{\mathrm{~d} t}=-r E_{y}-j_{y}+r A_{0} \cos \Omega t=-\frac{\partial \Phi}{\partial E_{y}}+r A_{0} \cos \Omega t,\left.\quad E_{y}\right|_{t=0}=E_{y 0},
$$

where $\Phi$ is the potential function $\Phi$ - the synergetic potential (generalized function of entropy production $[6-8]$ )

$$
\Phi\left(E_{y}, E_{x}, T, r, H\right)=\frac{1}{2} r E_{y}^{2}+\int_{0}^{E_{y}} j_{y}\left(E_{y}^{\prime}, E_{x}, T, H\right) d E_{y}^{\prime}
$$

and

$$
A_{0}=\frac{U_{0}}{L E_{0}}, \quad r=\frac{R_{c p}}{R}, \quad R_{c p}=\frac{L E_{0}}{S j_{0}},
$$

where the value of $R_{c p}$ has the meaning of sample resistance in weak electric fields.

Consider first the unforced system $\left(A_{0}=0\right)$. According to methods of catastrophe theory $[17,18]$, the asymptotic $(t \rightarrow \infty)$ solutions of the problem (3.3) are identified as critical values of the order parameters $E_{y}^{c r}$ of the potential function $\Phi$.

The parametric representation of critical points is defined implicitly by the equation of system state

$$
\frac{\partial \Phi}{\partial E_{y}}=r E_{y}+j_{y}\left(E_{y}, \boldsymbol{c}\right)=0 \Leftrightarrow E_{y}^{c r}=E_{y}^{c r}(\boldsymbol{c}),
$$

where $\boldsymbol{c}=\left\{r, E_{x}, T, H\right\}$ is the vector of control parameters. The (multiple-valued) function $E_{y}^{c r}(\boldsymbol{c})$ defines the catastrophe manifold constructed by critical points of potential $\Phi$.

The stability of critical values with respect to small fluctuations may be defined by the Morce characteristic of potential $\Phi$ - the critical curvature $[17,18]$

$$
W^{c r}(\boldsymbol{c})=\left.\frac{\partial^{2} \Phi}{\partial E_{y}^{2}}\right|_{E_{y}=E_{y}^{c r}}=r+\left.\frac{\partial j_{y}}{\partial E_{y}}\right|_{E_{y}=E_{y}^{c r}} .
$$

At $W^{c r}>0$ the critical point $E_{y}^{c r}$ defines the stable state of the system (macrostate), and at $W^{c r}<0$, the unstable one. Under the criticality condition $W^{c r}=0$ the critical point is degenerate.

The static macrostates of a system may be obtained as asymptotic $(t \rightarrow \infty)$ solutions of the problem (3.3) at given values of control parameters and for the unforced system $A_{0}=0$ :

$$
E_{y}^{s t}(\boldsymbol{c})=\lim _{t \rightarrow \infty} E_{y}(t)=E_{y}^{c r}(\boldsymbol{c}) \quad \text { at } A_{0}=0 \text { and } W^{c r}(\boldsymbol{c})>0 .
$$


Thus, the set of all macrostates in a space $\left\{\left(E_{y}^{s t}(\boldsymbol{c}), \boldsymbol{c}\right)\right\} \subset R^{1} \otimes R^{4}$ represents the attractor of the dynamical system (3.3). The boundaries of attraction regions define the unstable states (repeller) and are defined as $E_{y}^{c r}(\boldsymbol{c})$ at $W^{c r}(\boldsymbol{c})<0$.

At fixed values of control parameters the phase trajectory $E_{y}(t)$ tends, as $t \rightarrow \infty$, to some branch of attractor depending on the initial value $E_{y 0}$, i.e., the macrostate $E_{y}^{s t}$ may depend on its initial value in a discontinuous way. Thus, at some values of control parameters the system is characterized by multistability. Under variation of control parameters, crises of attractor branches take place, leading to bifurcations and nonequilibrium phase transitions (NPTs), which are interpreted as structural reorganizations.

The bifurcation set (local separatrix) in the control parameter space $\left(r, E_{x}, T, H\right)$ is defined by the condition of degeneration of critical points $\left(W^{c r}=0\right)$ and may be determined by elimination of parameter $E_{y}$ from the conditions

$$
\frac{\partial \Phi}{\partial E_{y}}\left(E_{y}, \boldsymbol{c}\right)=r E_{y}+j_{y}=0, \quad \frac{\partial^{2} \Phi}{\partial E_{y}^{2}}\left(E_{y}, \boldsymbol{c}\right)=r+\frac{\partial j_{y}}{\partial E_{y}}=0 .
$$

Whenever the control parameters vary very slowly, the system is quasi-static and follows the convention of perfect delay $[17,18]$. Thus, if some path in control parameter space traverses the local separatrix, the reorganization of a system macrostate, the NPT, occurs. The local separatrix is a set of confluence points at which the potential $\Phi$ is not a Morce function and is structurally unstable. By transversal crossover of the local separatrix due to continuous change of parameters the number of static states changes by 2 , one of them being the macrostate. At some direction of traverse some local minimum and maximum become confluent and a degenerate stationary state occurs which disappears under further change of the parameters. Under reverse change of the parameters two stationary states appear "from nothing". Thus, the local separatrix separates the parameter space into open regions in which the number of macrostates is constant and every point $\boldsymbol{c}=\left(E_{x}, T, H, r\right)$ parameterizes the structurally stable potential function of a qualitatively different type.

Here some computational problems had to be solved. The main one is connected with the necessity to solve the nonlinear equation (3.6) to get numerically the parametric representation of critical values $E_{y}^{c r}$ and calculate the corresponding $W^{c r}$. Because the function $E_{y}^{c r}(\boldsymbol{c})$ may be multiple-valued and the number of its functional branches is not known in advance, the use of traditional methods of Newton type leads to the necessity to accomplish prolonged numerical experiments to identify all regions of branching and appropriate functional branches. In addition, traditional numerical optimization methods are poorly adapted to handle situations where the objects under study (in this case, the synergetic potential and its derivatives) are given not analytically, but by an array of numerical data obtained as a result of the preliminary numerical solution of some other problem (e.g., the problem (2.12)). Similar problems arise in simulations of a bifurcation set by solving the nonlinear system (3.9). Because of the large number of variables, some will be considered as fixed. A typical investigation problem is to find diagrams where one of the control parameters $x$ changes, provided that the other parameters, $\boldsymbol{c} \backslash\{x\}$, are fixed.

To solve these problems, we have used our (computer-oriented) numerical method based on ideas of cluster analysis (hierarchy classification), which we call a direct search algorithm. Omitting the details of programming, the main ideas of this algorithm are the following.

Consider the problem of solving the equation $F(x, y)=0$ with an additional condition of any type, e.g., with the condition $G(x, y)>0$. All points which satisfy the equation $F(x, y)=0$ form a level curve on the $(x, y)$ plane. To get the parameter representation $y=y(x)$, define

RUSSIAN JOURNAL OF NONLINEAR DYNAMICS, 2021, 17(2), 141-156 
the grid values of variables $x_{i}$ and $y_{j}$ and get the set of knot cells which covers a given region of the $(x, y)$ plane. By looking over all sides of knot cells, find those of them which are intersected by a given level curve and define the intersection points by inverse interpolation. If the intersection point satisfies the additional condition, then place it to the two-connected list. Thus, the basic list of points

$$
S=\left\{\left(x_{i}, y_{i}\right) \mid F\left(x_{i}, y_{i}\right) \approx 0, G\left(x_{i}, y_{i}\right)>0\right\}
$$

will be formed.

At this preliminary step of the algorithm the selected feasible points are disordered on the $(x, y)$ plane. To simulate the branches $y=y(x)$, define some metric $\rho(x, y)$ on the set $\left\{\left(x_{i}, y_{i}\right)\right\}$ (we have used the Euclidean distance) and some limiting value $\rho_{\max }$ (we have used the maximal cell diameter). Sort the basic list $S$ on increasing $x_{i}$ 's. Form the first supplementary list (the first cluster) in the following way. Exclude the first element from the basic list and put it into a given cluster. Sort the remaining points of the basic list on the distance (in the sense of metric $\rho$ ) from the last point of the given cluster and find in the basic list the point which is the nearest. If its (the smallest) distance is larger than $\rho_{\max }$, then the first cluster is formed. Otherwise exclude the found nearest element from the basic list and put it into the given cluster as a second successive point. Repeat the procedure for the second cluster point and so on until the formation of first cluster is finished. If the basic list is not empty, form the second cluster in a similar way and so on. The procedure being finished, the set of feasible points (3.10) will be decomposed into a set of nonintersecting clusters $S^{(k)}, k=1, \ldots$ Each cluster represents the list of successive points with ordered variables $\left\{\left(x_{j}^{(k)}, y_{j}^{(k)}\left(x_{j}\right)\right)\right\}, j=1,2, \ldots$ and simulates a separate functional branch $y^{(k)}=y^{(k)}(x)$ on the $(x, y)$ plane. Thus, the set of all clusters simulates the (multiple-valued) solution of the equation $F(x, y)=0$ with the given additional condition. Data from the list $S^{(k)}$ may be used for calculation of any functional $\mathcal{F}(x)=\mathcal{F}\left(y^{(k)}(x), x\right)$ at any connected component of solutions of the given problem.

The algorithm is of great advantage since it can deal with any model simulation in which some nonlinear equation with possible additional conditions must be solved and works equally well in situations where no analytical expression for $F(x, y)$ is available (e.g., if the set $F\left(x_{i}, y_{j}\right)$ is obtained by some numerical procedure). All branches defined implicitly by the equation $F(x, y)=0$ including isolated subsets are obtained at once without any initial approximations. In addition, by using our method the critical (turning or branching) points manifest themselves in a natural way and need no special algorithms for their computation and investigation. Our method also allows one to take into account the feasibility conditions, i.e., to form the lists of points with desired properties (e.g., the equilibrium or critical points with a selected type of stability) and investigate them separately. The accuracy may easily be controlled due to the choice of grid points. Numerical experiments have demonstrated a complete agreement with the known model analytical solutions.

The same ideas were implemented for the procedure for excluding the parameter $y$ from the system of two nonlinear equations $F_{1}\left(y, x_{1}, x_{2}\right)=0, F_{2}\left(y, x_{1}, x_{2}\right)=0$, which defines an implicit (multi-valued) function $x_{1}=x_{1}\left(x_{2}\right)$. The $y$ parameter is excluded as follows. Each of the equations defines a one-parameter family of zero-level lines in the plane $\left(y, x_{1}\right)$, depending on the parameter $x_{2}$. Given $x_{2}=x_{2 i}=f i x$, the system defines two zero-level lines on the plane $\left(x_{1}, y\right)$. At given grid values of the variables $x_{1}$ and $y$ the set of intersection points $\left\{\left(x_{1 i j}, y_{i j}\right)\right\}$ of these lines can be obtained. After that, the clustering procedure described above can be used to obtain three-dimensional branches. Eliminating the parameter $y$ can be achieved by projecting these branches onto the $\left(x_{1}, x_{2}\right)$ plane. 
The algorithms described above were encoded by using the object-oriented means of $\mathrm{C}++$ programming language. Despite the simplicity of the ideas underlying the above algorithms (which essentially come down to a direct search for the necessary points), their software implementation required a fairly sophisticated use of object-oriented programming tools provided by $\mathrm{C}++$. The developed program is a robust and quick tool for computer simulation of critical manifolds and bifurcation sets in situations of multiple branching and was effectively used for analysis of bifurcations of equilibrium states in highly nonlinear systems [6-8, 13, 14, 19]. All diagrams presented below in figures were constructed with the help of this direct search algorithm.

The results of computer simulation of a bifurcation set are presented in Fig. 2. In Fig. 2a the icons show the behavior of synergetic potential $\Phi$ at marked points. The local extreme points of $\Phi$ define the critical values of $E_{y}^{c r}$ and the local minima correspond to macrostates. The presented part of the $\left(E_{x}, T\right)$ plane is decomposed into open regions, denoted as 1, 2, 3 and 4, in which potential $\Phi$ has 1, 2, 3 and 4 minima, respectively (the system has an appropriate number of macrostates).

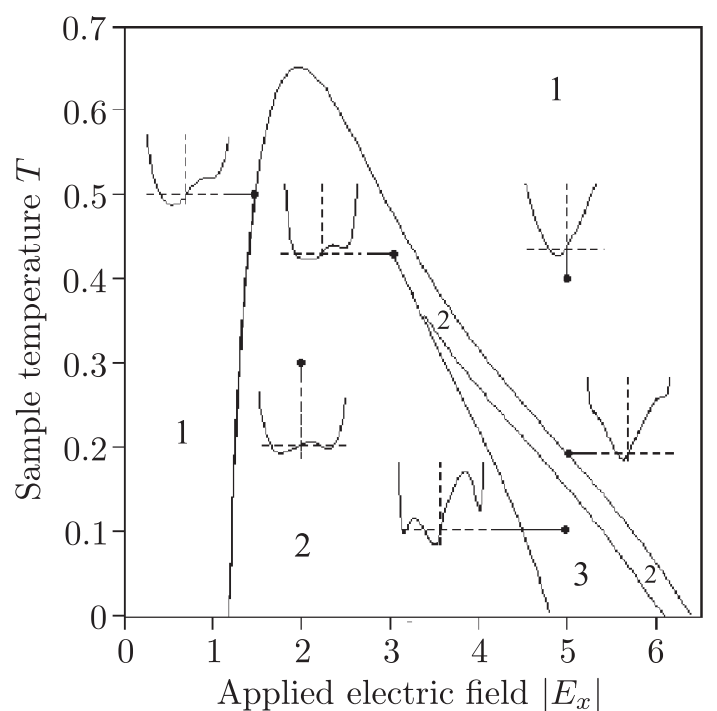

(a)

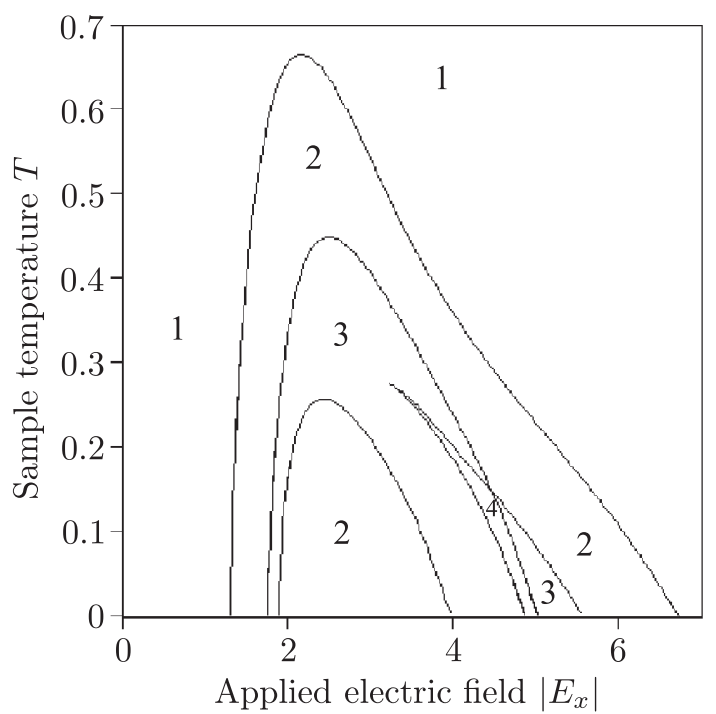

(b)

Fig. 2. Simulation results: the local separatrix on the control parameters plane $\left(E_{x}, T\right)$ for $r=0.04$ : (a) $H=0.3$; (b) $H=1$.

Note that in the presence of a magnetic field the system exhibits a fractal-like bifurcation set. Each region of multi-stability on the control parameter plane includes cusp-shaped "Arnold tongues" of higher multistability. As some path on the control parameters plane traverses the "Arnold tongues" of high multistability, a large number of fuzzy bifurcations occur.

The results of simulations of the critical transverse field under the conditions $H=0$ and $H=0.3 ; 1$ are presented in Fig. 3 .

The region $E_{x}<1$ attracts attention because, on the one hand, in the absence of a magnetic field we have $E_{y}^{s t}=0$ and, on the other hand, the Hall effect appears unusually (the field $E_{y}^{s t}$ has a maximum and changes sign). The presence of a nonzero magnetic field plays a principal role because it smears out the phase transitions at turning points $E_{1}$ and $E_{2}$ and forces the system to make a completely determinate selection between two symmetric conditions which are of equal probability at $H=0$. Thus, the magnetic-field-induced symmetry breaking takes place. 
At small $H=0.3$ for $E_{x}>1$ the situation is close to the "inoculating" one $(H=0)$, which is quite natural, because the magnetic field is weak and the appropriate corrections are small (Fig. 3a).

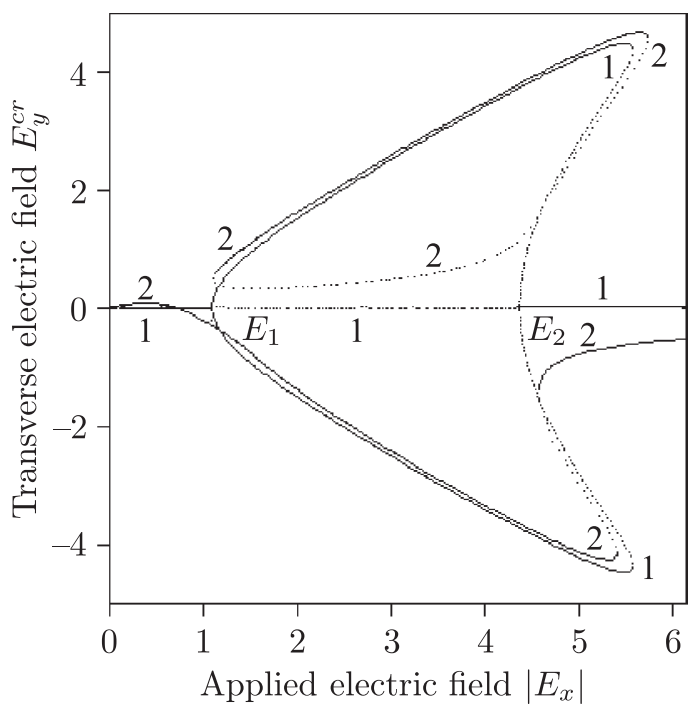

(a)

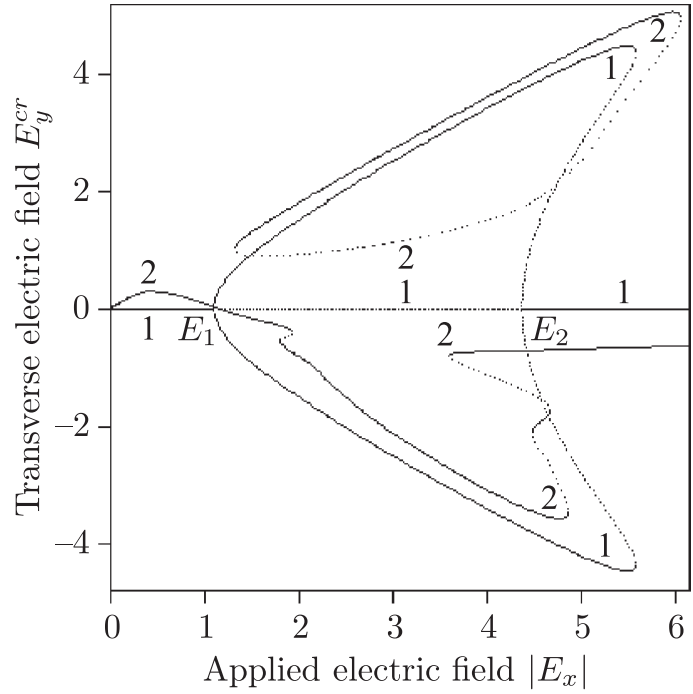

(b)

Fig. 3. Simulation results: bifurcation diagrams or a critical transverse field (3.6) for $r=0.04, T=0.1$ depending on $E_{x}$. Curves 1 correspond to $H=0$. Curves 2 correspond to: (a) $H=0.3$; (b) $H=1$. The solid parts of the curves represent the macrostates (attractor), and the dotted parts indicate the unstable states (repeller). At $H>0$ the symmetry breaking of static macrostates is observed.

Similar effects appear when one changes a parameter $T$ at $E_{x}=$ const as shown in Fig. 4a, where the critical transverse field (3.6) depending on sample temperature $T$ is presented.

At $H=0$ the bifurcation diagram is symmetrical and demonstrates the effect of applied electric-field-induced ferroelectricity of quasi-two-dimensional electron gas [7]. At $H=1$ the "Curie temperature" point $T_{1}$ is smeared out by the magnetic-field-induced forced bifurcation.

The bifurcation diagrams demonstrate one of the universal properties of nonlinear systems the existence of "chaotic" regimes which can be viewed globally in the whole parameter space, where the bifurcation scheme is found to possess a much more complex hierarchical structure and coexists with the macrostates exhibiting multiple hysteresis behaviors [20].

The potential profiles demonstrate similar peculiarities if the kinetic regimes are located close to the high multistability "tongues". Accordingly, under conditions of high multistability the fine structure of potential profiles exhibits fuzzy extreme points, as shown in Fig. 4b. In fact, at $H=1$ and $E_{y}<0$ three macrostates in the plateau region merge into one, and even a small uncertainty in control parameters yields a substantial change in the macrostate of the system. This means that an extraordinary high accuracy of control parameters is required for a reliable prediction of the eventual macrostate. We call such situation a macrostate crisis [8], which may be interpreted as the system being at the onset of chaos.

Here we may note that at crisis parameters the system becomes "neutral-stable", which means that in the presence of external noise a point on the critical manifold can execute a random work and thus cause a large fluctuation. 


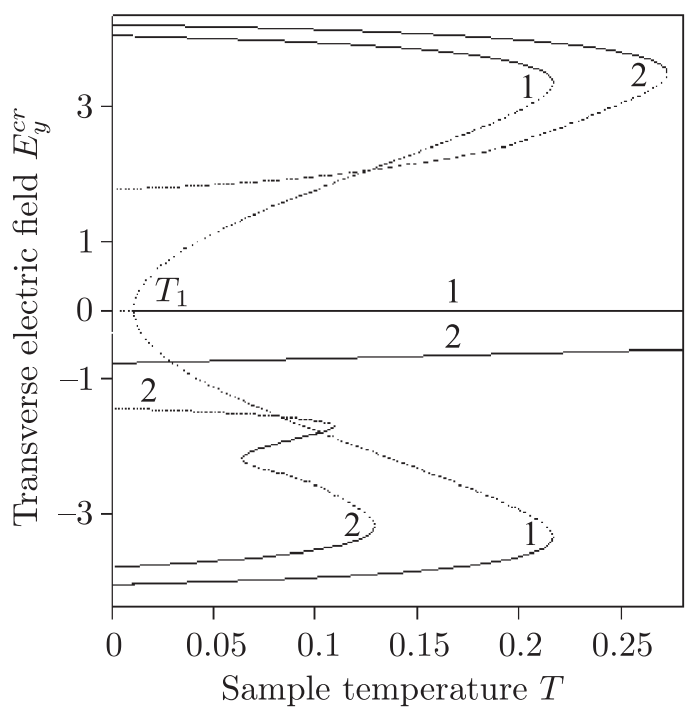

(a)

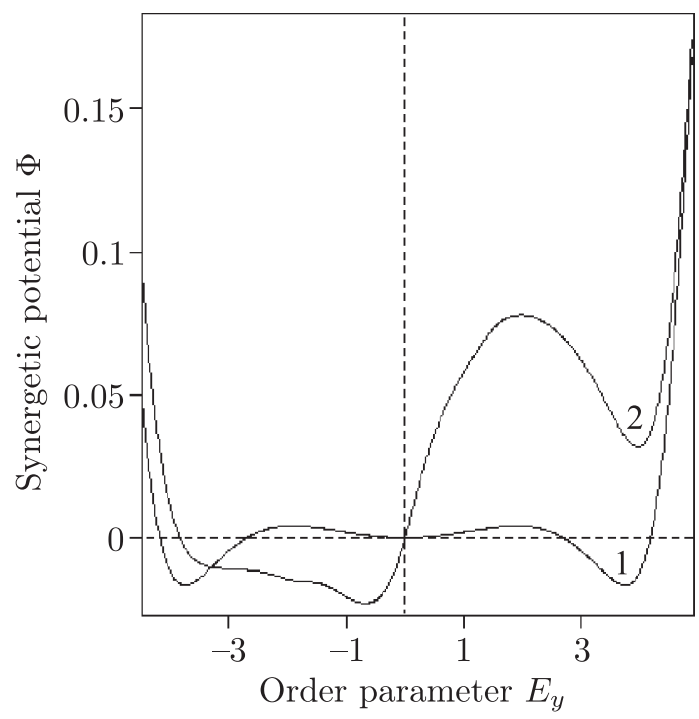

(b)

Fig. 4. Simulation results: (a) Critical transverse field (3.6) for $r=0.04, E_{x}=4.64$ depending on $T$ for $H=0$ (curves 1) and $H=1$ (curves 2). The solid parts of the curves represent the macrostates (attractor), and the dotted parts indicate the unstable states (repeller). Existence of nonzero branches means that at some values of control parameters the nonequilibrium electron gas exhibits ferroelectric properties. (b) Potential profiles for $H=0$ (curve 1) and $H=1$ (curve 2) at $r=0.04, E_{x}=4.5$, $T=0.15$. Curve 2 contains a plateau region at $E_{y}<0$ with three mild minima.

\section{Harmonic forcing and generation of even harmonics}

At $A_{0} \neq 0$ the deterministic equation (3.3) describes the evolution of the order parameter under harmonic forcing. For the moment we may view the system as a "black box" and look at how the output power and waveform change through the mechanism of controlled bifurcations as the input signal and system parameters are varied.

After the transient processes are over, the system tends to a steady state regime, when periodic external influence leads to periodic (nonlinear) fluctuations $E_{y}^{a s}(t)$ of the order parameter around the equilibrium position.

We use the harmonic studies to quantify the distortion in power and current waveforms in various kinetic regimes in the system under study using the decomposition of system output in terms of harmonics. Harmonics have a physical interpretation and an intuitive appeal.

Thus, we present a system response in the form of Fourier series

$$
E_{y}^{a s}(t)=\bar{E}_{y}^{a s}+\sum_{k=1}^{\infty} A_{k} \cos \left(k \Omega t-\varphi_{k}\right)
$$

Here the dc value $\bar{E}_{y}^{a s}$, the amplitude $A_{k}$ and the phase shift $\varphi_{k}$ of the $k$ th harmonic component are estimated numerically as described in $[9,10]$.

When passing the input signal through the nonlinear system, the spectrum of the output process is enriched. If the input signal is harmonic, then the periodic modulation of the system leads to periodic perturbations of the potential and of the corresponding stationary microstates. 
As a result, higher harmonics are registered in the spectrum of output signal power. The amplification coefficient at the $k$ th harmonics due to nonlinearities is characterized by the ratio

$$
\eta_{k}=\left(\frac{A_{k}}{A_{0}}\right)^{2}=Q_{k}^{2}
$$

where $Q_{k}=A_{k} / A_{0}$ is the amplitude response [21].

Define $f(t)=E_{y}^{a s}(t)-\bar{E}_{y}^{a s}$, when the dc component is subtracted from the output signal. Then the output is called a hidden half-wave symmetry if

$$
f(t)=-f\left(t+\frac{T_{\Omega}}{2}\right), \quad T_{\Omega}=\frac{2 \pi}{\Omega} .
$$

The spectrum of a signal with hidden half-wave symmetry contains only odd harmonics (in the case of symmetric potential, i.e., at $H=0$ [9]).

Our goal is to illustrate the effect of output signal half-wave symmetry breaking (resulting in the appearance of even harmonics) induced by the magnetic field.

For numerical simulations let us define the values of controlled parameters at which the effects of even harmonics generation occur. We have confirmed the simulation results within the adiabatic approximation for small driving frequencies $\Omega \ll 1$. All calculations in this section were accomplished at $\Omega=0.001$.

Figure 5 demonstrates that the static magnetic field violates the hidden half-wave symmetry of the output signal and leads to the appearance of even harmonics.

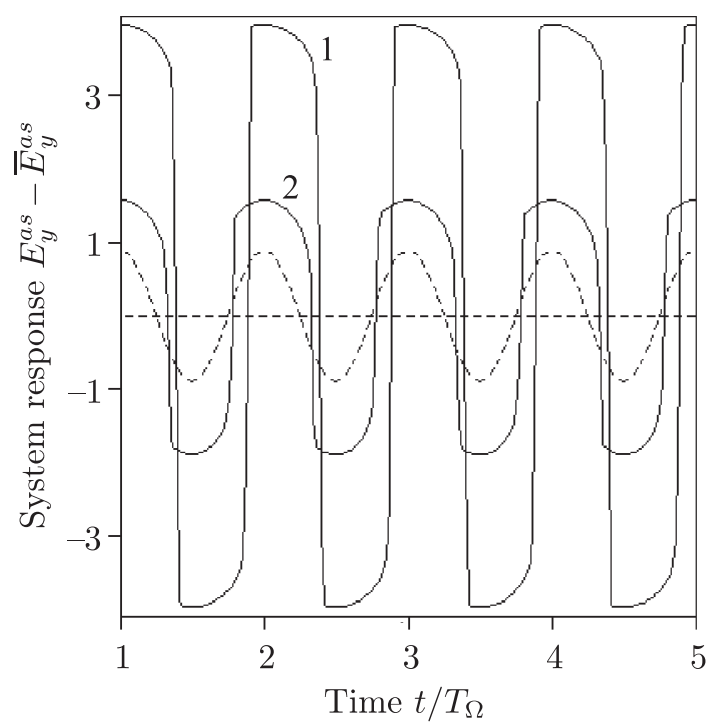

(a)

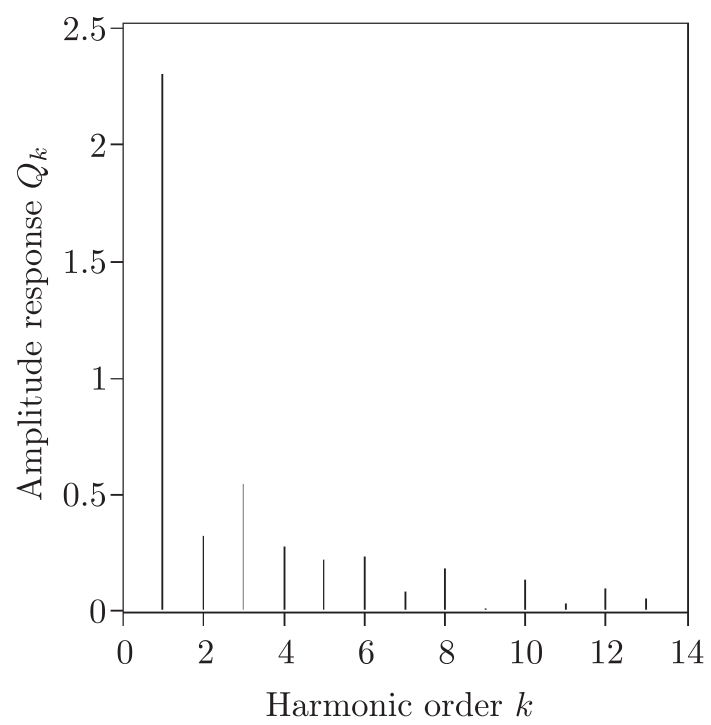

(b)

Fig. 5. Simulation results for $r=0.04, T=0.15, E_{x}=4.5, A_{0}=0.9$ : (a) system response for different magnetic fields. The dashed curve is the input signal $A_{0} \cos \Omega t$, curve 1 is the system output signal at $H=0$ (the hidden half-wave symmetry is observed, so even harmonics are absent), curve 2 is the system output signal at $H=1$ and contains even harmonics in addition to odd ones (see Fig. 5b); (b) harmonic (amplitude) spectrum for $H=1$. Magnitudes of even harmonics (which are absent at $H=0$ ) are compatible with higher odd ones.

The effect of even harmonics generation has a distinct energetic activation barrier in the form of a threshold with respect to changing the input voltage $A_{0}$ (Fig. 6a). Magnitudes of harmonics can be controlled by the magnetic field $H$ (Fig. 6b). 


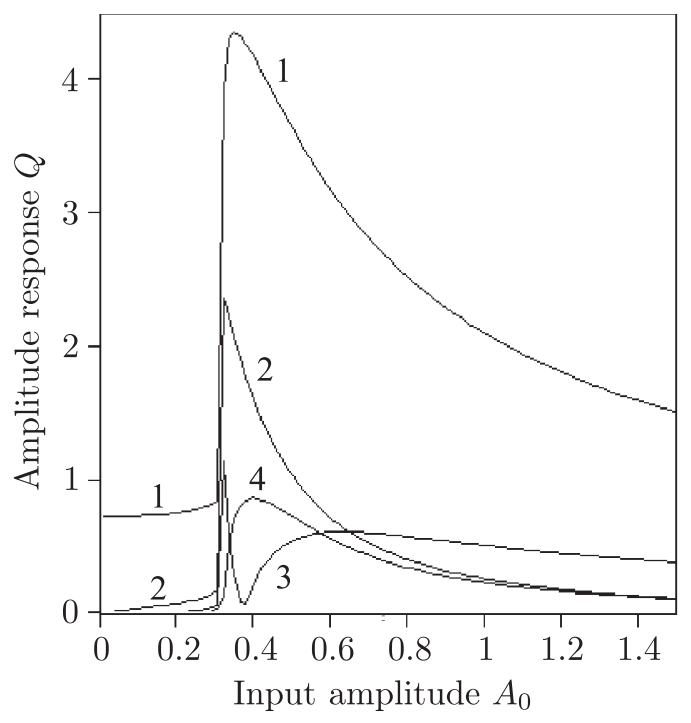

(a)

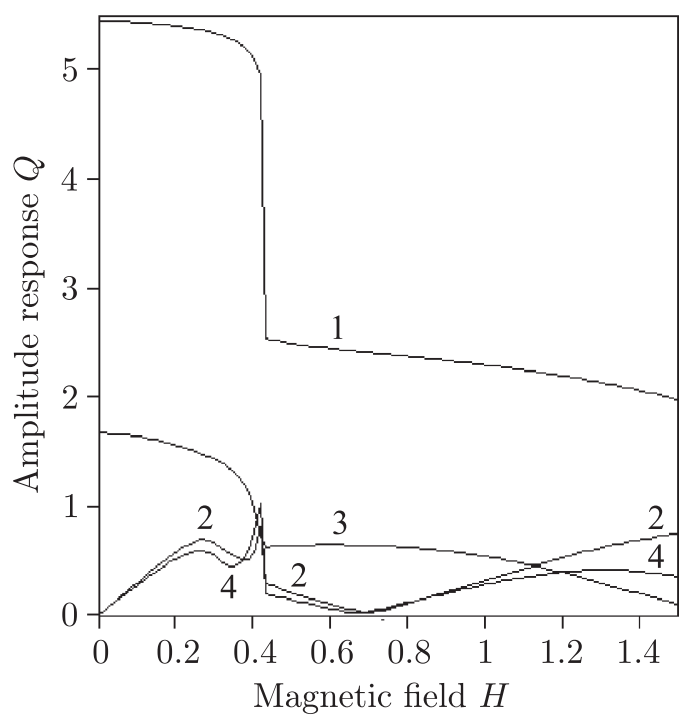

(b)

Fig. 6. Simulation results: amplitude response for the first four harmonics at $r=0.04, T=0.15$, $E_{x}=4.5$. (a) $H=1$. Lines $1,2,3$ and 4 represent $Q_{1}, Q_{2}, Q_{3}$ and $Q_{4}$, respectively, depending on $A_{0}$. (b) $A_{0}=0.9$. Lines $1,2,3$ and 4 represent $Q_{1}, Q_{2}, Q_{3}$ and $Q_{4}$, respectively, depending on $H$.

Figure 7 shows that magnitudes of higher order harmonics can be controlled by the applied field $E_{x}$ or by sample temperature $T$. The curves demonstrate resonant behaviors with maxima near the points of the local separatrix.

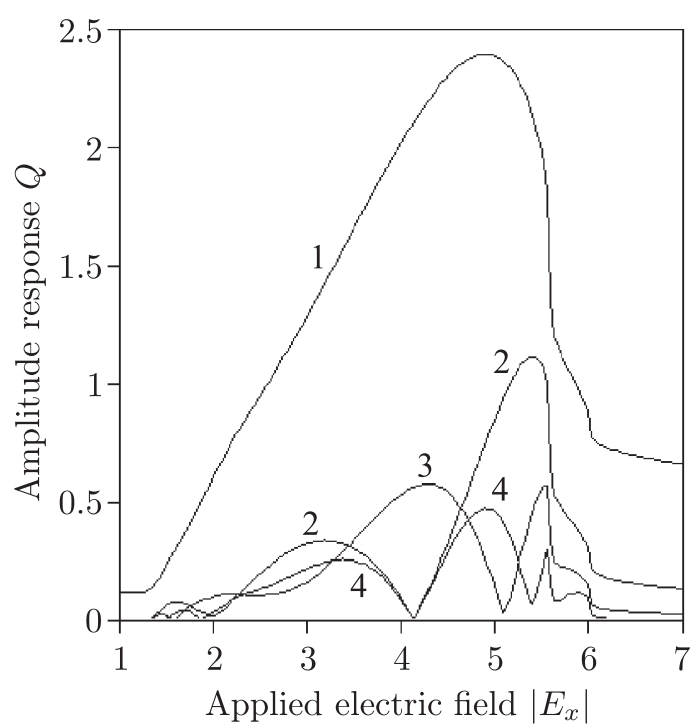

(a)

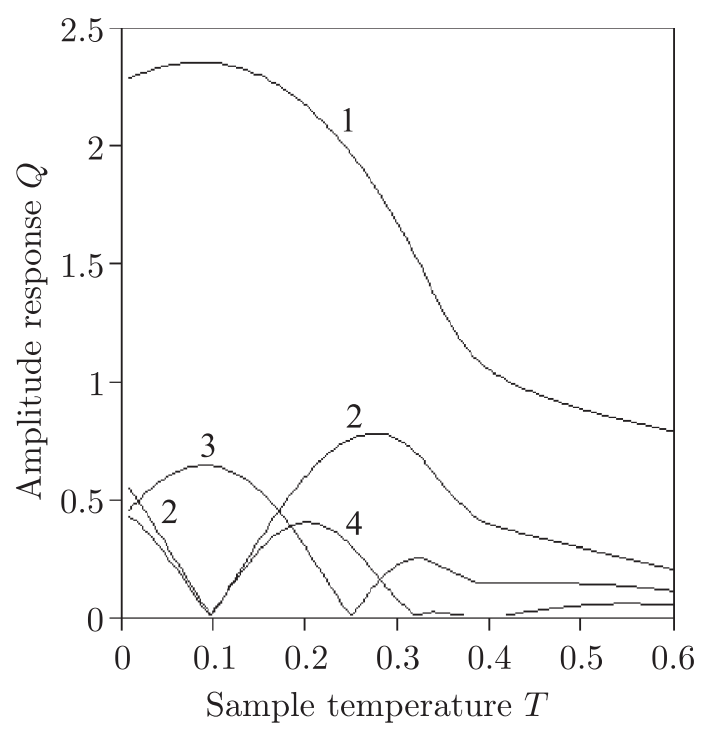

(b)

Fig. 7. Simulation results: amplitude response for the first four harmonics at $r=0.04, H=1, A_{0}=0.9$. (a) $T=0.15$. Lines $1,2,3$ and 4 represent $Q_{1}, Q_{2}, Q_{3}$ and $Q_{4}$, respectively, depending on $E_{x}$. (b) $E_{x}=4.5$. Lines $1,2,3$ and 4 represent $Q_{1}, Q_{2}, Q_{3}$ and $Q_{4}$, respectively, depending on $T$. 


\section{Discussion}

In summary, our analysis has discovered the novel phenomena of bifurcations of static macrostates and the parameter-tuning effect of even harmonics generation in the presence of a static magnetic field when viewed from the nonequilibrium phase transitions in lateral superlattices. As model simulations show, under then action of a harmonic forcing signal one can expect a rich structure of behaviours of output response of quasi-two-dimensional electron gas. An even harmonics generation detection strategy, based on bifurcation-based principles, is further proposed and explained in detail by exploring the influences of system output response with different input signals.

The underlying mechanism requires the following basic ingredients of a system: (i) a coherent input (such as a periodic signal); (ii) a self-organization mechanism that is inherent in the system and that adds to the coherent input. (iii) an energetic activation barrier or, more generally, a form of threshold. Given these features, the response of the system undergoes resonance-like behavior of magnitudes of odd and even harmonics as a function of control parameters. This is not strictly a resonance in the sense of an increased response when a driving frequency is tuned to the "natural frequency" of the system. In our system we observed the resonant behaviours when output response is maximized when some parameters - in this case the applied field, the sample temperature and the magnetic field - are tuned near certain values, namely, the points of symmetry breaking.

Here, some important aspects are summarized and related to computer simulations.

1. By variation of inner control parameters we change the form and number of extreme points of the potential function which governs the dynamics of the system, thus controlling the reorganizations of the macrostates of the unforced system (bifurcations).

2. In the presense of a magnetic field complex nonlinear behaviors including high multistability of static macrostates and hysteresis behaviors are discovered. By enlarging the magnetic field successively, the nonlinear behaviors show self-organized hierarchical complexity. A self-similar fine structure of the bifurcation set appears ("Arnold tongues"), showing fractal-like behavior, which leads to fractal-like folding of critical manifold sheets, resulting in fuzzy bifurcations.

3. In order to achieve finer and finer structure, we require an exhausting time of computation. Usually, with limited resolution and time, the fine structure is smeared out, many regions of higher multistability are too narrow to detect.

4. The physical mechanism responsible for even harmonics generation is provided by symmetry violation of static macrostates and is sustained by a constant magnetic field.

5. The appearance of even harmonics in the system output manifests itself through the breaking of the hidden half-wave symmetry of the output signal.

6. A unique synergetic potential model makes it more efficient and effective to adjust the system parameters at which the effect of even harmonics generation comes up more easily. The most sensitive are the symmetry breaking points, at those values of control parameters for which a degeneration of the potential function occurs.

7. We have demonstrated the electric and temperature control of the output response of quasi-two-dimensional electron gas under harmonic forcing and observed the resonant behaviors when even harmonics of output signal power are maximized when some parameters - in this case the applied field and sample temperature - are tuned near certain values. The maximal manifestation of the amplification effect occurs along the local separatrix. 
Numerical estimations of the effects considered are reduced, in general, to measurement units. At $d=10^{-8} \mathrm{~m}, \tau=10^{-12} \mathrm{~s}, \Delta=1.6 \cdot 10^{-21} \mathrm{~J}, n=10^{21} \mathrm{~m}^{-3}$ we get $T_{0} \approx 100 \mathrm{~K}$, $E_{0} \approx 6.6 \cdot 10^{4} \mathrm{~V} / \mathrm{m}, j_{0} \approx 2.4 \cdot 10^{7} \mathrm{~A} / \mathrm{m}^{2}, H_{0} \approx 4.3 \cdot 10^{5} \mathrm{~A} / \mathrm{m}, \Omega_{0} \approx 10^{13} \mathrm{~s}^{-1}$.

\section{Conclusions}

The investigations of nonequilibrium phase transitions may lead to a more profound understanding of physical mechanisms of instabilities in semiconductor structures and may reveal the role that the properties and parameters of materials play in these phenomena. Investigations in the region of self-organization open up possibilities for the creation of fundamentally new physical devices.

The great interest shown in recent decades in the generation of higher-order harmonic frequencies has been due to a wide range of their possible applications in science and technology.

The parameter-tuning high harmonics generation through bifurcations holds great promise for the application of signal processing in nonlinear communications systems. In this paper, we report, for the first time to our knowledge, a theoretical and numerical study of the possibility of controlled even harmonic generation through controlled bifurcation by quasi-two-dimensional electron gas in the presence of a static perpendicularly applied magnetic field.

This paper investigates the implementation of a new bifurcation-based model to realize the thermally and electrically controlled even harmonics generation effect. This model is based on advanced materials such as lateral superlattices and presents an interesting scenario in which the self-organization effects contribute significantly to the occurrence of even harmonics generation through bifurcation, a situation that is not found in most familiar models.

Here we offer the first detailed study of the frequency response of lateral superlattices, as well as a method designed to find the values of system parameters that result in intense even harmonics generation. The crux of the method is to locate regions in control parameter space where the output response is intensified. These findings are supported by extensive numerical simulations.

The results of this paper demonstrate that the interplay of external driving and self-organization leads to the onset of specific spectral features in the output power spectra of nonlinear systems. Thus, investigations in the region of self-organization open up possibilities for the creation of fundamentally new physical devices. We believe that the simple and generic principles underlying the phenomenon considered suggest its applicability to a wide variety of physical systems.

\section{References}

[1] Guckenheimer, J. and Holmes, Ph., Nonlinear Oscillations, Dynamical Systems, and Bifurcations of Vector Fields, 5th ed., Appl. Math. Sci., vol. 42, New York: Springer, 1997.

[2] Arnold, V.I., Afrajmovich, V.S., Il'yashenko, Yu.S., and Shil'nikov, L. P., Bifurcation Theory and Catastrophe Theory, Encyclopaedia Math. Sci., vol. 5, Berlin: Springer, 1999.

[3] Morozov, A. Yu. and Reviznikov, D. L., Modeling of Dynamic Systems with Interval Parameters in the Presence of Singularities, Russian J. Nonlinear Dyn., 2020, vol. 16, no. 3, pp. 479-490.

[4] Jafrate, G. J., Ferry, D., and Reich, R., Lateral (Two-Dimensional) Superlattices: Quantum-Well Confinement and Charge Instabilities, Surf. Sci., 1982, vol. 113, nos. 1-3, pp. 485-488.

[5] Reich, R.K., Crondin, R. O., and Ferry, D. K., Transport in Lateral Surface Superlattices, Phys. Rev. B, 1983, vol. 27, no. 6, pp. 3483-3493. 
[6] Shmelev, G. M. and Maglevanny, I. I., Transverse EMF in Lateral Superlattices, Physics of LowDimensional Structures, 1996, vol. 9-10, pp. 81-88.

[7] Epshtein, E. M., Shmelev, G. M., and Maglevanny, I. I., Ferromagnetic and Ferroelectric Properties of Nonequilibrium Electron Gas, Phys. Lett. A, 1999, vol. 254, nos. 1-2, pp. 107-111.

[8] Maglevanny, I. I., Highly Nonlinear Phenomena of Self-Organization of Quasi-Two-Dimensional Electron Gas in High Magnetic and Electric Fields, Phys. Status Solidi B, 2009, vol. 246, no. 6, pp. 12971305.

[9] Maglevanny, I. I., Smolar, V. A., and Karyakina, T. I., Thermally and Electrically Controllable Multiple High Harmonics Generation by Harmonically Driven Quasi-Two-Dimensional Electron Gas, Superlattices Microstruct., 2018, vol. 118, pp. 29-44.

[10] Maglevanny, I. I., Smolar, V.A., and Karyakina, T. I., Weak Signals Amplification through Controlled Bifurcations in Quasi-Two-Dimensional Electron Gas, Russian J. Nonlinear Dyn., 2018, vol. 14, no. 4, pp. 453-472.

[11] Grahn, H. T., von Klitzing, K., Ploog, K., and Döhler, G.H., Electrical Transport in NarrowMiniband Semiconductor Superlattices, Phys. Rev. B, 1991, vol. 43, no. 14, pp. 12094-12097.

[12] Bass, F. G. and Tetervov, A. P., High-Frequency Phenomena in Semiconductor Superlattices, Phys. Rep., 1986, vol. 140, no. 5, pp. 237-322.

[13] Shmelev, G. M., Epshtein, E. M., Maglevanny, I. I., and Yudina, A. V., Anomalous Hall Effect in Quasi-Two-Dimensional Superlattices in High Electric Field, Physics of Low-Dimensional Structures, 1996, vol. 4-5, pp. 47-55.

[14] Epshtein, E. M., Maglevanny, I. I., and Shmelev, G. M., Electric-Field-Induced Magnetoresistance of Lateral Superlattices, J. Phys. Condens. Matter, 1996, vol. 8, no. 25, pp. 4509-4514.

[15] Press, W.H., Teukolsky, S. A., Vetterling, W. T., and Flannery, B. P., Numerical Recipes: The Art of Scientific Computing, 3rd ed., Cambridge: Cambridge Univ. Press, 2007.

[16] Hairer, E., Nørsett, S.P., and Wanner, G., Solving Ordinary Differential Equations: Vol. 1. Nonstiff Problems, 2nd ed., Springer Ser. Comput. Math., vol. 8, New York: Springer, 1993.

[17] Gilmore, R., Catastrophe Theory for Scientists and Engineers, New York: Dover, 1993.

[18] Poston, T. and Stewart, I., Catastrophe Theory and Its Application, London: Pitman, 1978.

[19] Maglevanny, I. I., Meletlidou, E., and Stagika, G., Numerical Investigation of Bifurcations of Equilibria and Hopf Bifurcations in Disease Transmission Models, Commun. Nonlinear Sci. Numer. Simul., 2011, vol. 16, no. 1, pp. 284-295.

[20] Aoki, K., Nonlinear Dynamics and Chaos in Semiconductors, Boca Raton, Fla.: CRC, 2000.

[21] Landa, P.S. and McClintock, P. V.E., Vibrational Resonance, J. Phys. A, 2000, vol. 33, no. 45, pp. L433-L438. 